\title{
Risk factors associated with experienced stigma among people diagnosed with mental ill-health: a cross-sectional study
}

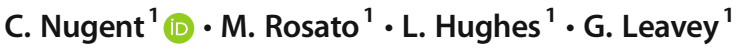 \\ Published online: 28 August 2020 \\ (C) The Author(s) 2020
}

\begin{abstract}
Purpose: to examine the relationship between religiosity, social support, trauma, quality of life and experienced stigma of mental illness amongst a population diagnosed with mental ill-health. Methods: A cross-sectional survey of day service users in Northern Ireland $(n=295)$ covering a range of issues including religiosity, social support, quality of life and prior experience of trauma. Stigma was measured using a recognised stigma scale. We used multinomial logistic regression to examine risk factors associated with experienced stigma. Results: Univariate analysis showed significant associations between stigma and age, number of friends, social support, quality of life and prior experience of trauma. Age, quality of life, and trauma remained independently associated with stigma in a multivariate logistic regression model $\left(\mathrm{x}^{2}(12)=98.40, p<0.001\right)$. Conclusion: Younger people, those with less social support, prior experience of trauma and with poorer quality of life are at increased risk of experiencing stigma related to their diagnosis of mental illness. The findings provide further understanding of stigma and are useful for those overseeing programmes to improve access to mental health treatment.
\end{abstract}

Keywords Mental illness · Stigma $\cdot$ Religiosity $\cdot$ Quality of Life $\cdot$ Trauma $\cdot$ Social Support

\section{Introduction}

Stigma and discrimination pose significant problems for people diagnosed with mental illness and may lead to real-life disadvantages, including poor access to mental and physical

\section{Nugent}

chris.nugent@hotmail.co.uk

L. Hughes

lynettehughes1@yahoo.ie

1 Bamford Centre for Mental Health and Wellbeing; School of Psychology, Ulster University, Cromore Road, Coleraine BT52 1SA, Northern Ireland 
healthcare [1, 2], problems gaining or maintaining employment [3], difficulty accessing accommodation [4] and reduced life-expectancy [5, 6]. Mental illness may also impact negatively on stigmatised individuals by inducing self-stigma, self-judgement and reinforcement of negative stereotypes [7]. These can trigger low self-esteem [8], a why-try effect and loss of confidence when pursuing personal goals [5]. Self-perception of stigma amongst people with mental illness often leads to poorer attitudes towards psychotherapy [6], or even avoidance of professional help-seeking $[9,10]$ which in turn may lead to prolongation of symptoms and ultimately worse outcomes, including increased mortality.

\section{Factors associated with Stigma}

Previous research suggests younger people report more mental illness-related stigma $[11,12]$, while those with higher levels of education are more likely to conceal their diagnosis [9]. Some studies show a gender effect but the evidence is inconsistent [10, 13-18]. Some evidence suggests a mediating role for social support, with increased peer support helping to challenge internalised stigma related to mental illness [19]. Conversely, a lack of support from public services or friends and family may be perceived as discrimination [20], while greater comfort in the ability to disclose mental illness to friends and family impacts positively on mental wellbeing [21]. Other studies have however found increased social support may be associated with a greater perception of stigma [22].

Similar ambiguity surrounds the impact of religiosity with some research [23] reporting the positive impact of religiosity on both physical and mental health, quality of life and life satisfaction, and another finding an association between religiosity and increased public stigma towards mental illness [24].

The relationship between trauma and stigma is well-established. Individuals exposed to traumatic events such as war or sexual abuse often feeling stigmatised because of their experiences, leading to feelings of shame and negatively impacting their ability to seek professional help. A recent study amongst refugee men in Australia for example found PTSD severity was associated (indirectly via mental health stigma) with lower help-seeking intentions [25]. Similarly, Amone-P'Olak et al [26], in their analysis of survivors of sexual violence during war in Northern Uganda found those reporting sexual abuse perceived more stigma and reported more barriers to seeking care than their peers without such experiences. Conversely, Andresen \& Blais [27] found higher self-stigma was associated with a lower likelihood of disclosing military sexual trauma among female veterans.

People who attend day centres may be at increased risk of experiencing mental illnessrelated stigma. Their attendance at the centre makes it more difficult to conceal their diagnosis and may be itself associated with stigma independent of a diagnosis of mental illness. This population have been traditionally neglected in stigma research and little is known about the factors that may exacerbate or protect them against the negative effects of the stigma that surrounds their diagnosis of mental illness.

This study examines the relationship between religiosity, social support, trauma, quality of life and experienced stigma of mental illness amongst a population diagnosed with mental illhealth who attend a day centre in Northern Ireland. 


\section{Method}

\section{Sample}

Stratified sampling was used to select participants with mental health problems $(n=504)$ within day centres across Northern Ireland (NI) $(n=14)$. Of those selected, $61 \%$ consented to participate $(n=306)$ and were provided with information sheets. Ethical approval was granted by the Office for Research Ethics Committees Northern Ireland (ORECNI). All data was anonymised prior to analysis.

\section{Data Collection}

A comprehensive questionnaire was designed for use in the study and included questions related to socio-demographic characteristics and religiosity. The questionnaire also included established data-collection instruments - the Stigma Scale [28]; the Lancashire Quality of Life Profile (LQoLP) [29]; the Medical Outcomes Study (MOS) Social Support Survey [30]; and the Brief Trauma Questionnaire (BTQ) [31].

\section{Instruments}

Stigma was measured using the Stigma Scale [28], a twenty-eight item five-point Likert-type questionnaire designed to measure stigma associated with mental illness as perceived by service users, it includes components on positive and negative aspects of stigma, and internalization. Scores (global stigma) range from 0 to 112 with higher scores indicating more feelings of stigma and includes three subscales- discrimination, disclosure and putative positive aspects of mental illness. The scale was originally tested in a North London population of people diagnosed with mental illness and was shown to have both good internal reliability (Chronbach's $\alpha=0.87$ ) and test-retest reliability ( $\kappa$ coefficients ranged from $0.4-$ $0.71)$.

\section{Quality of Life}

The LQoLP [29] is a 105-item measure of Quality of Life using a seven-point scale (where higher scores indicate better QoL/satisfaction). It measures global well-being along nine life domains - work, leisure, religion, finance, living situation, legal and safety issues, family relations, social relations and health. An overall Quality of Life score was generated by taking an average of scores on each of the subscales.

Social Support was measured using the MOS Social Support Survey [30], a 20-item measure covering four dimensions - emotional/informational, tangible, affection and positive social interaction - and a summary overall functional social support index. For this study only the overall index was used - with scores ranging from one to five (from lower to higher social support respectively).

Trauma was measured using eight items from the Brief Trauma Questionnaire (BTQ) [31], a ten-item questionnaire measuring exposure to traumatic experiences, including violence towards the participant or a family member, unwanted sexual contact, involvement in a serious accident, and suffering a life-threatening illness. Items measuring exposure to war or war- 
related injuries and major natural or technological disasters were excluded. Scores range from zero to eight with higher scores reflecting greater exposure to traumatic events.

\section{Data Analysis}

All analysis was carried out in Stata 13 [32]. Participants aged over seventy-five years $(n=6)$ and those for whom age was unknown $(n=5)$ were excluded from analysis. Missing values in the responses to the twenty-eight items of the Stigma Scale were imputed using the Stata $\mathrm{Mi}$ command [35, [33]. A summary stigma score (0-122) was generated by summing these items, and further summarised into four evenly distributed categories $(0-46,47-56,57-66$ and 67112) - very low, low, medium and high respectively.

Descriptive statistics summarised the demographic characteristics of the sample, and logistic regression examined their association with stigma. Significantly associated variables $(\mathrm{p}<=0.05)$ were then included in a final multinomial logistic regression analysis (using the Stata mlogit macro): in these analyses independent variables no longer retaining significance in the fully adjusted model were dropped, and a subsequent model estimated.

\section{Results}

After exclusions 295 people participated. Table 1 shows the age and gender breakdown by levels of stigma. Mean age was 51.8 years $(\mathrm{SD}=10.2$, range $=23-73)$ and $52 \%$ were female $(n=153)$. Global stigma scores ranged from 20 to 95 (mean $=56.6$, $\mathrm{SD}=13.7)$ with females scoring higher than males (mean $=57.2, \mathrm{SD}=13.8$ and 55.7, $\mathrm{SD}=13.6$ respectively) and those in the youngest age-group scoring highest (mean = 63.7, $\mathrm{SD}=16.32$ ). Stigma scores generally decreased with increasing age: for males $53 \%$ and $5 \%$ reported high stigma levels in the youngest and oldest age groups respectively; and similarly, for females $47 \%$ and $12 \%$ reported high levels of stigma. Table 2 shows $16 \%(n=47)$ of participants were very religious, 53\% $(n=155)$ reported being single or unmarried and $51 \%(n=129)$ reported having five or more close friends and/or relatives. Scores on the social support index ranged from 1 to 5 with a mean of $3.36(\mathrm{SD}=0.92)$, overall Quality of Life scores ranged from 1.987.00 (mean $=5.16, \mathrm{SD}=0.77$ ) and scores on the BTQ ranged from 0 to 8 with a mean of $1.96(\mathrm{SD}=1.79)$.

Table 1 Global Stigma Scale scores by stigma category, gender and age group

\begin{tabular}{|c|c|c|c|c|c|c|c|c|}
\hline \multirow[t]{2}{*}{ Stigma Score } & \multicolumn{4}{|c|}{ Males $(n=142)$} & \multicolumn{4}{|c|}{ Females $(n=153)$} \\
\hline & $\begin{array}{l}23-39 \\
\%(n)\end{array}$ & $40-49$ & $50-59$ & $60+$ & $\begin{array}{l}23-39 \\
\%(\mathrm{n})\end{array}$ & $40-49$ & $50-59$ & $60+$ \\
\hline Very Low $(0-46)$ & $21(4)$ & $23(10)$ & $26(11)$ & $35(13)$ & $13(2)$ & $19(8)$ & $16(9)$ & $39(16)$ \\
\hline Low $(47-56)$ & $5(1)$ & $16(7)$ & 37 (16) & $41(15)$ & $20(3)$ & $17(7)$ & $25(14)$ & $27(11)$ \\
\hline Medium (57-66) & $21(4)$ & $33(14)$ & $19(8)$ & $19(7)$ & $20(3)$ & $36(15)$ & $27(15)$ & $22(9)$ \\
\hline $\operatorname{High}(67-112)$ & $53(10)$ & $28(12)$ & $19(8)$ & $5(2)$ & $47(7)$ & $29(12)$ & $31(17)$ & $12(5)$ \\
\hline Total & 19 & 43 & 43 & 37 & 15 & 42 & 55 & 41 \\
\hline
\end{tabular}


Table 2 Sociodemographic and other characteristics of the sample

\begin{tabular}{llc}
\hline Characteristics & Category & $\%(\mathrm{n})$ \\
\hline Age & $23-39$ & $11.5(34)$ \\
& $40-49$ & $28.8(85)$ \\
& $50-59$ & $33.2(98)$ \\
Gender & $60+$ & $26.4(78)$ \\
Religiosity & Female & $51.9(153)$ \\
Marital Status & Very Religious & $16.3(47)$ \\
& Married/Co-habiting & $20.5(60)$ \\
& Single/Unmarried & $52.9(155)$ \\
Number of Friends & Divorced/Separated & $21.8(64)$ \\
MOS Social Support Index & Widowed & $4.5(14)$ \\
Quality of Life & Five or more & $50.8(129)$ \\
BTQ Trauma & Mean (SD) & $3.36(0.92)$ \\
\hline
\end{tabular}

\section{Health and socio-psychological factors related to stigma}

Table 3 shows results of a univariate logistic regression analysis comparing those recording the higher levels of stigma with those reporting very low levels. Generally, older people were less likely to report low $(\mathrm{RR}=1.00,95 \% \mathrm{CI}=0.97,1.04)$, medium $(\mathrm{RR}=0.96,0.92,0.99)$ and high $(\mathrm{RR}=0.93,0.89,0.96)$ stigma levels than those younger. Females were associated with increased risks of reporting medium $(57-66)(\mathrm{RR}=1.38,0.72,2.64)$ or high stigma $(67-112)$ $(\mathrm{RR}=1.39,0.73,2.67)$, though the effects were not statistically significant. Participants who were very religious $(n=47)$ were less likely to report a low stigma score $(R R=0.46,0.15$, 1.43), and increased likelihood of reporting a high score $(R R=1.99,0.84,4.71)$. However, neither were statistically significant. With marital status - when compared to those married/cohabiting, all other groups recorded higher likelihoods for reporting stigma: those widowed had a $31 \%$ excess risk $(0.31,5.62)$ of reporting a medium stigma score while all other groups displayed decreased risk. Conversely, those widowed displayed a decrease in risk of reporting a high stigma score while all other groups showed an increase. Those single/unmarried showed the greatest increases in risk $(\mathrm{RR}=1.66,0.66,4.16)$ but again no result was statistically significant. Having five or more close friends or relatives had a protective effect across all stigma levels - risk of reporting a medium stigma score was reduced by $\sim 60 \%$ ( $R R=0.39$, $0.19,0.81)$ and risk of reporting a high score by $76 \%(\mathrm{RR}=0.24,0.11,0.50)$ compared to those with less than five close friends/relatives.

The Social Support Index showed a clear relationship with stigma: when compared to very low stigma levels, higher levels showed an inverse relationship with social support - $\mathrm{RR}=$ 0.80: $95 \% \mathrm{CI}=0.55,1.16$; $\mathrm{RR}=0.56,0.39,0.82$; and $\mathrm{RR}=0.55: 0.38,0.79$ for low, medium and high levels respectively. Quality of Life scores also significantly reduced the association with stigma. Each unit increase in Quality of Life was associated with a reduced risk of experiencing stigma. When compared to very low stigma levels $-\mathrm{RR}=0.45: 95 \% \mathrm{CI}=0.26$, 0.79 (low); $\mathrm{RR}=0.27: 0.16,0.48$ (medium) and $\mathrm{RR}=0.14: 0.08,0.25$ (high stigma). Finally, those with a greater history of trauma (BTQ) experienced more stigma: compared with very low stigma levels, a greater history of trauma were associated with increases of $46 \%$ (RR = $1.46,1.18,1.80)$ and $69 \%(\mathrm{RR}=1.69,1.36,2.01)$ in medium and high levels of stigma respectively. 
Table 3 Univariate analysis of stigma by category and independent variables

\begin{tabular}{|c|c|c|c|c|}
\hline \multirow[t]{2}{*}{ Independent Variable } & & \multicolumn{3}{|l|}{ Stigma $^{\wedge}$} \\
\hline & & $\begin{array}{l}\text { (Very low vs) Low } \\
\text { RR }(95 \% \mathrm{CI})\end{array}$ & $\begin{array}{l}\text { (V. low vs) Medium } \\
\text { RR }(95 \% \text { CI) }\end{array}$ & $\begin{array}{l}\text { (V. low vs) High } \\
\text { RR }(95 \% \mathrm{CI})\end{array}$ \\
\hline Age & Continuous & $1.00(0.97,1.04)$ & $0.96(0.92,0.99)^{* *}$ & $0.93(0.89,0.96)^{* * *}$ \\
\hline Gender & Females & $0.97(0.51,1.86)$ & $1.38(0.72,2.64)$ & $1.39(0.73,2.67)$ \\
\hline Religiosity & Very & $0.46(0.15,1.43)$ & $1.57(0.66,3.78)$ & $1.99(0.84,4.71)$ \\
\hline \multirow[t]{4}{*}{ Marital Status } & Married/Co-habiting & 1.00 (ref) & 1.00 (ref) & 1.00 (ref) \\
\hline & Single/Unmarried & $0.64(0.28,1.47)$ & $0.85(0.36,1.99)$ & $1.66(0.66,4.16)$ \\
\hline & Divorced/Separated & $0.74(0.28,1.95)$ & $0.88(0.32,2.37)$ & $1.31(0.45,3.84)$ \\
\hline & Widowed & $0.35(0.06,2.18)$ & $1.31(0.31,5.62)$ & $0.70(0.11,4.59)$ \\
\hline Number of Friends & Five or more & $0.48(0.23,1.00)$ & $0.40(0.19,0.81)^{*}$ & $0.24(0.11,0.50)^{* * * *}$ \\
\hline MOS Social Support & Continuous & $0.80(0.55,1.16)$ & $0.56(0.39,0.82)^{* *}$ & $0.55(0.38,0.79)^{* *}$ \\
\hline Quality of Life & Continuous & $0.45(0.26,0.79)^{* * *}$ & $0.27(0.16,0.48)^{* * * *}$ & $0.14(0.08,0.25)^{* * *}$ \\
\hline BTQ Trauma & Continuous & $1.02(0.81,1.27)$ & $1.46(1.18,1.80)^{* * *}$ & $1.69(1.36,2.01)^{* * *}$ \\
\hline
\end{tabular}

${ }^{\wedge}$ Very Low $(0-46)$ category is reference group

$* \mathrm{P}=<0.05, * * \mathrm{P}=<0.01, * * * \mathrm{P}=<0.001$

\section{Multivariate Analysis}

Indicators associated with stigma at univariate level $(\mathrm{p}<=0.05)$ were included in the multivariate analysis (Table 4). In each case Model 1 (M1) shows the fully adjusted model. Quality of life and trauma remained statistically significant predictors of stigma after adjusting for age, gender, number of friends, social support and each other. Older participants were associated with a small risk reduction $(5 \%)$ for reporting high levels of stigma $(\mathrm{RR}=0.95,95 \% \mathrm{CI}=0.91$, 0.99 ) while those reporting good quality of life showed a risk reduction of around $84 \%$ (RR = $0.16,0.08,0.35)$. Increased exposure to traumatic events was associated with an excess risk of $39 \%$ for reporting high levels of stigma $(\mathrm{RR}=1.39,1.07,1.81)$.

Table 4 Multinomial logistic regression models of associated independent variables on relative risk of stigma per category compared to very low stigma $(0-46)$

\begin{tabular}{|c|c|c|c|c|c|c|}
\hline \multirow[t]{2}{*}{ Included Variables } & \multicolumn{2}{|c|}{ Low (47-56) } & \multicolumn{2}{|c|}{ Medium (57-66) } & \multicolumn{2}{|c|}{ High (67-112) } \\
\hline & $\begin{array}{l}\text { M1 } \\
\text { RRR }(95 \% \\
\text { CI })\end{array}$ & $\begin{array}{l}\text { M2 } \\
\text { RRR }(95 \% \\
\text { CI })\end{array}$ & $\begin{array}{l}\text { M1 } \\
\text { RRR }(95 \% \\
\text { CI })\end{array}$ & $\begin{array}{l}\text { M2 } \\
\text { RRR }(95 \% \\
\text { CI })\end{array}$ & $\begin{array}{l}\text { M1 } \\
\text { RRR }(95 \% \\
\text { CI) }\end{array}$ & $\begin{array}{l}\text { M2 } \\
\text { RRR }(95 \% \\
\text { CI })\end{array}$ \\
\hline Age & $\begin{array}{c}1.01(0.97 \\
1.05)\end{array}$ & $\begin{array}{c}1.01(0.97 \\
1.04)\end{array}$ & $\begin{array}{c}0.98(0.94 \\
1.02)\end{array}$ & $\begin{array}{c}0.97(0.93 \\
1.00)\end{array}$ & $\begin{array}{c}0.95(0.91 \\
0.99)^{*}\end{array}$ & $\begin{array}{c}0.94(0.90 \\
0.98)^{* *}\end{array}$ \\
\hline Gender (female) & $\begin{array}{c}0.64(0.31 \\
1.33)\end{array}$ & $\begin{array}{c}0.88(0.45 \\
1.73)\end{array}$ & $\begin{array}{c}1.15(0.53 \\
2.49)\end{array}$ & $\begin{array}{c}1.28(0.64 \\
2.58)\end{array}$ & $\begin{array}{c}1.26(0.55 \\
2.88)\end{array}$ & $\begin{array}{c}1.29(0.60, \\
2.76)\end{array}$ \\
\hline $\begin{array}{l}\text { Number of Friends } \\
(5+)\end{array}$ & $\begin{array}{c}0.60(0.27 \\
1.31)\end{array}$ & & $\begin{array}{c}0.76(0.33 \\
1.75)\end{array}$ & & $\begin{array}{c}0.70(0.29, \\
1.73)\end{array}$ & \\
\hline Quality of Life & $\begin{array}{c}0.42(0.21 \\
0.85)^{*}\end{array}$ & $\begin{array}{c}0.42(0.24 \\
0.75)^{* *}\end{array}$ & $\begin{array}{c}0.31(0.15 \\
0.64)^{* *}\end{array}$ & $\begin{array}{c}0.34(0.19 \\
0.61)^{* * * *}\end{array}$ & $\begin{array}{r}0.16(0.08 \\
0.35)^{* * *}\end{array}$ & $\begin{array}{r}0.18(0.10 \\
0.34)^{* * *}\end{array}$ \\
\hline $\begin{array}{l}\text { MOS Social } \\
\text { Support Index }\end{array}$ & $\begin{array}{c}1.05(0.67 \\
1.66)\end{array}$ & & $\begin{array}{c}0.82(0.51 \\
1.33)\end{array}$ & & $\begin{array}{c}0.96(0.57 \\
1.62)\end{array}$ & \\
\hline BTQ Trauma & $\begin{array}{c}1.02(0.78 \\
1.32)\end{array}$ & $\begin{array}{c}0.96(0.76 \\
1.21)\end{array}$ & $\begin{array}{c}1.38(1.07 \\
1.77)^{*}\end{array}$ & $\begin{array}{c}1.30(1.04 \\
1.62)^{*}\end{array}$ & $\begin{array}{c}1.39(1.07 \\
1.81)^{*}\end{array}$ & $\begin{array}{c}1.37(1.08, \\
1.73)^{* *}\end{array}$ \\
\hline
\end{tabular}

$* \mathrm{P}=<0.05, * * \mathrm{P}=<0.01, * * * \mathrm{P}=<0.001$ 
To estimate a second model (M2) - variables non-significant in M1 were removed leaving age, gender, quality of life and trauma. Increasing age was associated with a further risk reduction compared with $\mathrm{M} 1(\mathrm{RR}=0.94,95 \% \mathrm{CI}=0.90,0.98)$ while females showed excess risk of reporting the highest level of stigma compared to males $(\mathrm{RR}=1.29,0.60,2.76)$, though this was not statistically significant. Those reporting better quality of life remained at less risk $(\mathrm{RR}=0.18,0.10,0.34)$ but the effect was slightly smaller than in the previous model, and similarly those reporting greater exposure to traumatic events had a greater risk of reporting high levels of stigma but less so than in M1 ( RR = 1.37, 1.08, 1.73). Comparison of the models showed little difference overall. Relative risks for individual variables change only marginally between M1 and M2, with the largest changes noted for; gender and low ( $R R=0.64$ to $R R=$ $0.88)$, and medium $(\mathrm{RR}=1.15$ to $\mathrm{RR}=1.28)$ stigma scores.

\section{Discussion}

People with mental illness require appropriate support to help manage their condition. Stigma associated with mental illness presents significant barriers to this and presents practical, reallife problems for people with mental illness - for example, in finding and keeping work [3], accessing accommodation [4] and preventing those with mental illness from seeking professional help [34]. This study examines the relationship between stigma associated with mental illness and selected demographic and psychosocial factors. The Stigma Scale [28] measured stigma as perceived by people diagnosed with a mental illness, providing insight into how stigma is experienced by those directly affected. Participants were mostly aged over forty years, unemployed and either single or unmarried. Those at highest risk of feeling stigmatised were younger, had fewer than five close friends/relatives, poorer social support, had suffered greater exposure to traumatic events and generally reported poorer quality of life.

While the literature around stigma and age is ambiguous the findings reported here - that younger people were more likely to report stigma than their older counterparts - are consistent with other studies $[11,17,28]$. Oshodi et al [12] suggests this may be explained by the increased importance placed on dating and intimate relationships by younger people who are more likely to be in the process of starting their own families. They also suggest that younger respondents are perhaps more likely to have experienced unfair treatment at work because of their illness, when compared with older respondents who may have already attained senior positions in their workplace. Patten et al [35] suggests that the experience of stigmatisation may diminish over time as people learn to cope.

Similar to other studies $[9,12,35,36]$, females displayed greater risk $(\sim 40 \%)$ of experiencing stigma (both medium and high levels) than males, although this was not statistically significant.

Increased social support and having more than five close friends or relatives were associated with decreased risk of experiencing stigma in the univariate analysis. This is consistent with a number of other studies including Rusch et al [21], Robinson et al [37] and Moore \& Ayers [22]- who found that use of internet forums by women with post-natal mental illness enabled them to disclose their illness to others. This raises an interesting question for future work in terms of the distinction between social support from peers with a diagnosis of mental illness themselves, and those without mental illness. In spite of this however, neither association held up when entered into the multivariate regression models. 
Previous experience of trauma was strongly associated with stigma and the finding remained statistically significant after adjusting for age, gender, social support and quality of life. This is consistent with previous studies [38,39], [40], although it is unclear from the current findings whether the stigma experienced by those with a previous exposure to trauma is related solely to their subsequent diagnosis of mental illness or to the traumatic event itself. A further analysis of stigma experienced as a direct result of trauma may help better understand this association.

A strong-negative association was found between quality of life and experience of stigma, similar to other studies; [44, [41-44]. It is not possible from this analysis to infer the direction of the observed association and it seems intuitive that either could precede the other. For example, it seems intuitive that being surrounded by less stigma (and better social support) helps ensure a better quality of life for people with a diagnosis of mental illness. Alternatively, it is possible that individuals who report less stigma may have a better quality of life to begin with and a more positive outlook as a result, mediating the amount of stigma that surrounds them. A similar question was posed by Holubova et al. as a result of their analysis of self-stigma and quality of life [45]. Perhaps key to the relationship between stigma and quality of life is the process of selfstigma, the internalising of stigmatised beliefs towards oneself. Both Hsiao et al [42] and Huang et al [46] highlight the mediating role of self-stigma through it's impact on family coherence and individual quality of life, although both also emphasise the context of culture.

\section{Limitations}

The study sample comprised a specific group of people diagnosed with mental illness who attended a day centre in Northern Ireland. Participants were mostly aged over forty years, unemployed and single or unmarried, making it difficult to measure differences across the full age spectrum and estimate the effects of socioeconomic circumstances and health inequalities. Stigma was as experienced by people diagnosed with mental illness and is not therefore a measure of stigmatising beliefs held by the general population. It may also be likely that, since study participants have actively sought help, they may represent those who experience less stigma or are less acutely impacted by it than people who avoid seeking help altogether. Thus, levels of reported stigma may underestimate that experienced by the wider population of people living with mental illness. Finally, the cross-sectional design makes it impossible to infer causation in any of the associations reported in this study.

\section{Conclusions}

People with mental illness are often stigmatised because of their diagnosis which can, in turn, lead to a number of real-world disadvantages and negative outcomes. Those who are younger, with fewer friends and less social support, and who have been exposed to past trauma, experience greater risk of being stigmatised, making it potentially more difficult for such individuals to access adequate support. They also experience poorer quality of life. Interventions and programmes aimed at improving access to mental health treatment should take into account the additional complexity of stigma among such groups. The study raises questions for future work- including a comparison of the experience of stigma across mental health diagnoses, and further examination of the relationship between stigma related to mental illness and stigma related to trauma - such as sexual abuse - for individuals who have experienced both. 
Open Access This article is licensed under a Creative Commons Attribution 4.0 International License, which permits use, sharing, adaptation, distribution and reproduction in any medium or format, as long as you give appropriate credit to the original author(s) and the source, provide a link to the Creative Commons licence, and indicate if changes were made. The images or other third party material in this article are included in the article's Creative Commons licence, unless indicated otherwise in a credit line to the material. If material is not included in the article's Creative Commons licence and your intended use is not permitted by statutory regulation or exceeds the permitted use, you will need to obtain permission directly from the copyright holder. To view a copy of this licence, visit http://creativecommons.org/licenses/by/4.0/.

\section{References}

1. Laursen TM, Munk-Olsen T, Nordentoft M, Mortensen PB. Increased mortality among patients admitted with major psychiatric disorders: a register-based study comparing mortality in unipolar depressive disorder, bipolar affective disorder, schizoaffective disorder, and schizophrenia. J Clin Psychiatry. Jun. 2007;68(6): 899-907.

2. Gissler M, Laursen TM, Ösby U, Nordentoft M, Wahlbeck K. Patterns in mortality among people with severe mental disorders across birth cohorts: a register-based study of Denmark and Finland in 1982-2006. BMC Public Health. 2013;13:834.

3. Stuart H. Mental illness and employment discrimination. Curr Opin Psychiatry. Sep. 2006;19(5):522-6.

4. Ozmen E, Ogel K, Aker T, Sagduyu A, Tamar D, Boratav C. Public attitudes to depression in urban Turkey. Soc Psychiatry Psychiatr Epidemiol. Dec. 2004;39(12):1010-6.

5. P. W. Corrigan, A. B. Bink, A. Schmidt, N. Jones, and N. Rüsch, "What is the impact of self-stigma? Loss of self-respect and the 'why try' effect" J. Ment. Health, vol. 8237, no. February, pp. 1-6, 2014.

6. Xu Z, Müller M, Heekeren K, Theodoridou A, Dvorsky D, Metzler S, et al. Self-labelling and stigma as predictors of attitudes towards help-seeking among people at risk of psychosis: 1-year follow-up. Eur Arch Psychiatry Clin Neurosci. 2016;266(1):79-82.

7. Ben-Zeev D, Young MA, Corrigan PW. DSM-V and the stigma of mental illness. J Ment Health. 2010;19(4):318-27.

8. Corrigan PW, Watson AC. The paradox of self stigma and mental illness. Clin Psychol Sci Pract. May 2002;9(1):35-53.

9. Üçok A, Brohan E, Rose D, Sartorius N, Leese M, Yoon CK, et al. Anticipated discrimination among people with schizophrenia. Acta Psychiatr Scand. 2012;125(1):77-83.

10. S. Griffiths et al., "Self-stigma of seeking treatment and being male predict an increased likelihood of having an undiagnosed eating disorder," International Journal of Eating Disorders, vol. 48, no. 6. pp. 775778, Sep-2015.

11. Sirey JA, Bruce ML, Alexopoulos GS, Perlick DA, Raue P, Friedman SJ, et al. Perceived Stigma as a Predictor of Treatment Discontinuation in Young and Older Outpatients With Depression. Am J Psychiatry. Mar. 2001;158(3):479-81.

12. Oshodi YO, Abdulmalik J, Ola B, James BO, Bonetto C, Cristofalo D, et al. Pattern of experienced and anticipated discrimination among people with depression in Nigeria: A cross-sectional study. Soc Psychiatry Psychiatr Epidemiol. 2014;49(2):259-66.

13. Clement S, Schauman O, Graham T, Maggioni F, Evans-Lacko S, Bezborodovs N, et al. What is the impact of mental health-related stigma on help-seeking? A systematic review of quantitative and qualitative studies. Psychol Med. 2015;45(1):11-27.

14. Sarkin A, et al. Stigma experienced by people using mental health services in San Diego County. Soc. Psychiatry Psychiatr. Epidemiol. 2015;50:747-56.

15. Falsafi T, Valizadeh N, Sepehr S, Najafi M. Application of a Stool Antigen Test To Evaluate the Incidence of Helicobacter pylori Infection in Children and Adolescents from Tehran, Iran. Clin Vaccine Immunol. 2005;12(9):1094-7.

16. Kalisova L, Michalec J, Hadjipapanicolaou D, Raboch J. Factors influencing the level of self-stigmatisation in people with mental illness. Int J Soc Psychiatry. Jun. 2018;64(4):374-80.

17. Sedlácková Z, Kamarádová D, Prásko J, Látalová K, Ocisková M, Ocisková M, et al. Treatment adherence and self-stigma in patients with depressive disorder in remission - A cross-sectional study. Neuro Endocrinol Lett. 2015;36(2):171-7.

18. Vrbová K, Kamarádová D, Látalová K, Ocisková M, Praško J, Mainerová B, et al. Self-stigma and adherence to medication in patients with psychotic disorders - Cross-sectional study. Neuroendocrinol Lett. 2014;35(7):645-52. 
19. Moore D, Ayers S. Virtual voices: social support and stigma in postnatal mental illness Internet forums. Psychol Heal Med. May 2017;22(5):546-51.

20. S. Hamilton et al., "Factors associated with experienced discrimination among people using mental health services in England,” J. Ment. Heal., vol. 8237, no. June 2017, pp. 1-9, 2016.

21. Rüsch N, Brohan E, Gabbidon J, Thornicroft G, Clement S. Stigma and disclosing one's mental illness to family and friends. Soc Psychiatry Psychiatr Epidemiol. 2014;49(7):1157-60.

22. Hadera E, Salelew E, Girma E, Dehning S, Adorjan K, Tesfaye M. Magnitude and Associated Factors of Perceived Stigma among Adults with Mental Illness in Ethiopia. Psychiatry J. 2019;2019:8427561.

23. H. G. Koenig and H. G "Religion, spirituality, and health: the research and clinical implications.," ISRN Psychiatry, vol. 2012, Dec. 2012.

24. Tzouvara V, Papadopoulos C. Public stigma towards mental illness in the Greek culture. J Psychiatr Ment Health Nurs. 2014;21:931-8.

25. Byrow Y, Pajak R, McMahon T, Rajouria A, Nickerson A. Barriers to Mental Health Help-Seeking Amongst Refugee Men. Int. J. Environ. Res. Public Health. 2019;16(15):2634.

26. K. Amone-P'Olak, A. Elklit, and S. B. Dokkedahl, "PTSD, Mental Illness, and Care Among Survivors of Sexual Violence in Northern Uganda: Findings From the WAYS Study," Psychological Trauma: Theory, Research, Practice, and Policy, vol. 10, no. 3, pp. 282-289, May-2017.

27. Andresen FJ, Blais RK. Higher Self-Stigma is Related to Lower Likelihood of Disclosing Military Sexual Trauma During Screening in Female Veterans. Psychol Trauma Theory Res Pract Policy. May 2018;11(4): 372-8.

28. King M, et al. The Stigma Scale: development of a standardised measure of the stigma of mental illness. $\mathrm{Br}$ J Psychiatry. 2012:248-54.

29. Oliver JP, Huxley PJ, Priebe S, Kaiser W. Measuring the quality of life of severely mentally ill people using the Lancashire Quality of Life Profile. Soc Psychiatry Psychiatr Epidemiol. Feb. 1997;32(2):76-83.

30. Sherbourne C, Stewart A. The MOS social support survey. Social science \& medicine. 1991. Soc Sci Med. 1991;32(6):705-14.

31. P. P. Schnurr, M. J. Vielhauer, and M. N. Findler, "BTQ: Brief Trauma Questionnaire,” vol. 1, no. 2006, pp. $1-3,2009$.

32. StataCorp, "Stata Statistical Software: Release 13." StataCorp LP, College Station, TX, 2013.

33. Alcock A. A Gentle introduction to Stata. College Station, Texas: A Stata Press Publication, StataCorp LP; 2016.

34. Ali K, Farrer L, Fassnacht DB, Gulliver A, Bauer S, Griffiths KM. Perceived barriers and facilitators towards help-seeking for eating disorders: A systematic review. Int J Eat Disord. 2017;50(1):9-21.

35. Patten SB, Williams JVA, Lavorato DH, Bulloch AGM, Charbonneau M, Gautam M, et al. Perceived Stigma among Recipients of Mental Health Care in the General Canadian Population Les stigmates perçus chez les bé né ficiaires de soins de santé mentale dans la population gé né rale canadienne. Can J Psychiatr. 2016;61(8):480-8.

36. Ando S, Yamaguchi S, Aoki Y, Thornicroft G. Review of mental-health-related stigma in Japan. Psychiatry Clin Neurosci. 2013;67(7):471-82.

37. Robinson KJ, Rose D, Salkovskis PM. Seeking help for obsessive compulsive disorder (OCD): a qualitative study of the enablers and barriers conducted by a researcher with personal experience of OCD. Psychol Psychother Theory Res Pract. Jun. 2017;90(2):193-211.

38. Stolzenburg S, Freitag S, Schmidt S, Schomerus G. Associations between causal attributions and personal stigmatizing attitudes in untreated persons with current mental health problems. Psychiatry Res. Nov. 2018;260:24-9.

39. Deitz MF, Williams SL, Rife SC, Cantrell P. Examining Cultural, Social, and Self-Related Aspects of Stigma in Relation to Sexual Assault and Trauma Symptoms. Violence Against Women. May 2015;21(5): 598-615.

40. Burns B, Grindlay K, Holt K, Manski R, Grossman D. Military sexual trauma among US servicewomen during deployment: A qualitative study. Am J Public Health. Feb. 2014;104(2):345-9.

41. Ow C-Y, Lee B-O. Relationships Between Perceived Stigma, Coping Orientations, Self-esteem, and Quality of Life in Patients With Schizophrenia. Asia Pacific J Public Heal. Mar. 2015;27(2):NP1932-41.

42. Hsiao C-Y, Lu H-L, Tsai Y-F. Effect of family sense of coherence on internalized stigma and health-related quality of life among individuals with schizophrenia. Int J Ment Health Nurs. 2017:138-46.

43. Huang W-Y, Chen S-P, Pakpour AH, Lin C-Y. The Mediation Role of Self-Esteem for Self-Stigma on Quality of Life for People With Schizophrenia: A Retrospectively Longitudinal Study. J. Pacific Rim Psychol. Feb. 2018;12:e10.

44. Sánchez J, Rosenthal DA, Tansey TN, Frain MP, Bezyak JL. Predicting quality of life in adults with severe mental illness: Extending the International Classification of Functioning, Disability, and Health. Rehabil Psychol. 2016;61(1):19-31. 
45. Holubova M, Prasko J, Ociskova M, Marackova M, Grambal A, Slepecky M. Self-stigma and quality of life in patients with depressive disorder: A cross-sectional study. Neuropsychiatr Dis Treat. 2016;12(12):267786.

46. Huang W-Y, Chen S-P, Pakpour AH, Lin C-Y. The Mediation Role of Self-Esteem for Self-Stigma on Quality of Life for People With Schizophrenia: A Retrospectively Longitudinal Study. J Pacific Rim Psychol. Feb. 2018;12:e10.

Publisher's Note Springer Nature remains neutral with regard to jurisdictional claims in published maps and institutional affiliations.

Christopher Nugent is an independent researcher affiliated with the Bamford Centre for Mental Health and Wellbeing, Ulster University, Coleraine, Northern Ireland.

Dr Michael Rosato is Senior Research Fellow in the Bamford Centre for Mental Health and Wellbeing. He has a degree in Social Sciences and post-graduate qualifications in Computer Science, Medical Demography and a $\mathrm{PhD}$ in Epidemiology. Current research interests include; mental health; health outcomes of ethnic minority populations (in E\&W and in the wider European setting); chronic disease epidemiology and, more generally, health inequalities.

Dr Lynette Hughes is an independent researcher affiliated to the Bamford Centre, Ulster University. She has published on suicide and mental health in Northern Ireland.

Gerry Leavey is a health services researcher. He is Director of the Bamford Centre for Mental Health and Wellbeing, Ulster University and Clinical Co-Lead on the Clinical Research Network - Mental Health, Northern Ireland (NI). He is also Deputy Director of the Administrative Data Research Centre -NI. 